Supplement of Atmos. Chem. Phys., 21, 8823-8843, 2021

https://doi.org/10.5194/acp-21-8823-2021-supplement

(c) Author(s) 2021. CC BY 4.0 License.

(c) (1)
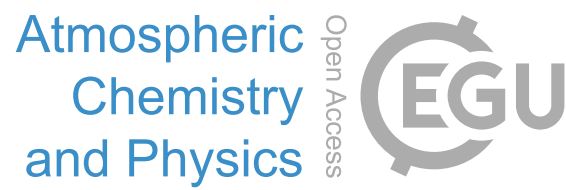

Supplement of

\title{
The middle atmospheric meridional circulation for 2002-2012 derived from MIPAS observations
}

\section{Thomas von Clarmann et al.}

Correspondence to: T. von Clarmann (thomas.clarmann@ kit.edu)

The copyright of individual parts of the supplement might differ from the article licence. 


\begin{abstract}
In this supplement the figures of the main paper are shown in colour scales which are hoped to be better suited for those who do not have a standard perception of color. The numbers of the figures are the same as in the main paper.
\end{abstract}



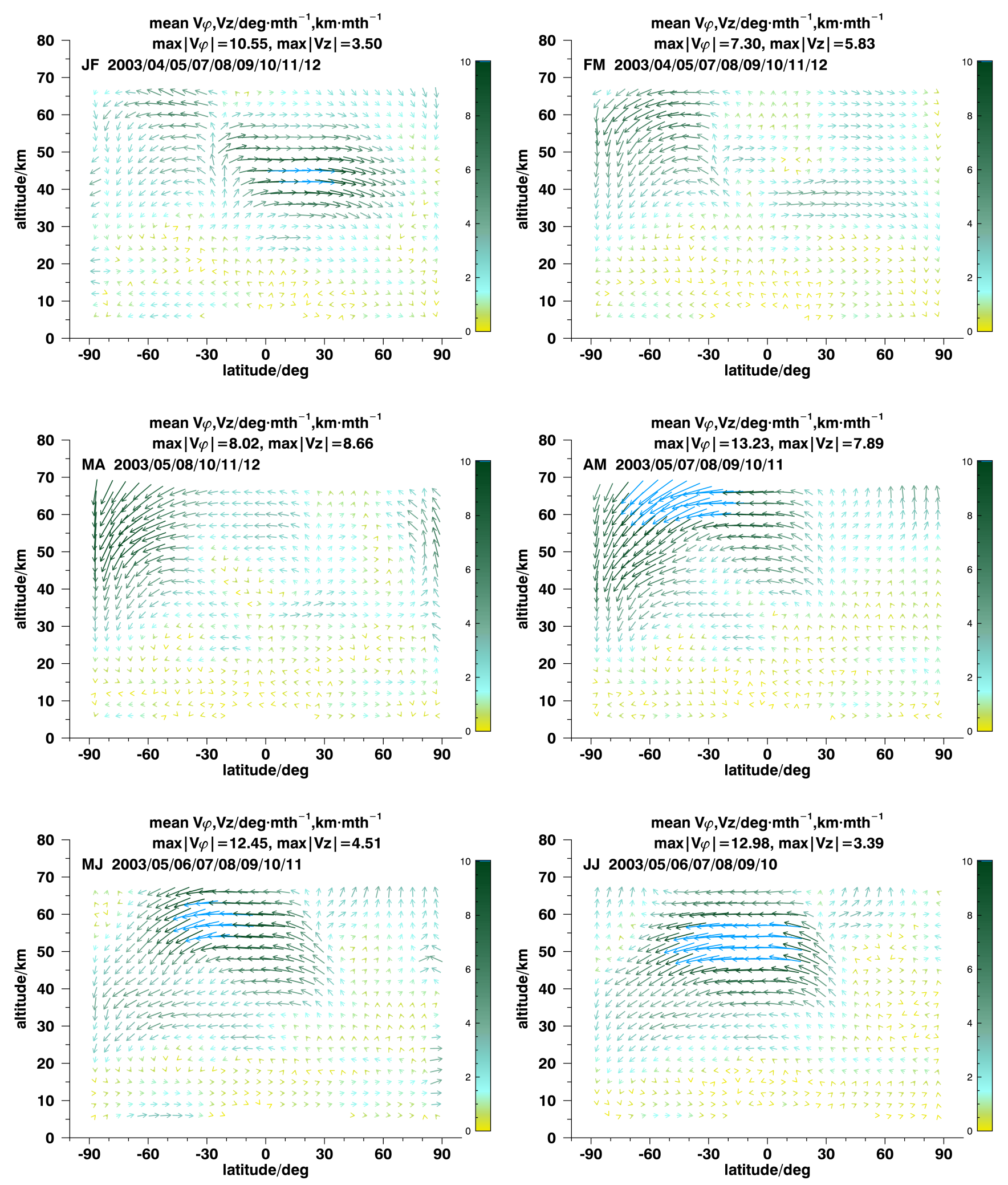

Figure S1: Mean monthly circulation patterns from January-February (top left, JF) to June-July (bottom right, JJ). The headers give quantitative information about maximal effective velocities, the months and years considered. Missing years are due to MIPAS data gaps and non-converged inversions. The colour scales refer to $\sqrt{\left(v_{\phi} \text { degree }{ }^{-1} \text { month }\right)^{2}+\left(v_{\mathrm{z}} \mathrm{km}^{-1} \text { month }\right)^{2}}$ for $v_{\phi}$ and $v_{\mathrm{z}}$ in units of degrees per month and kilometer per month. 

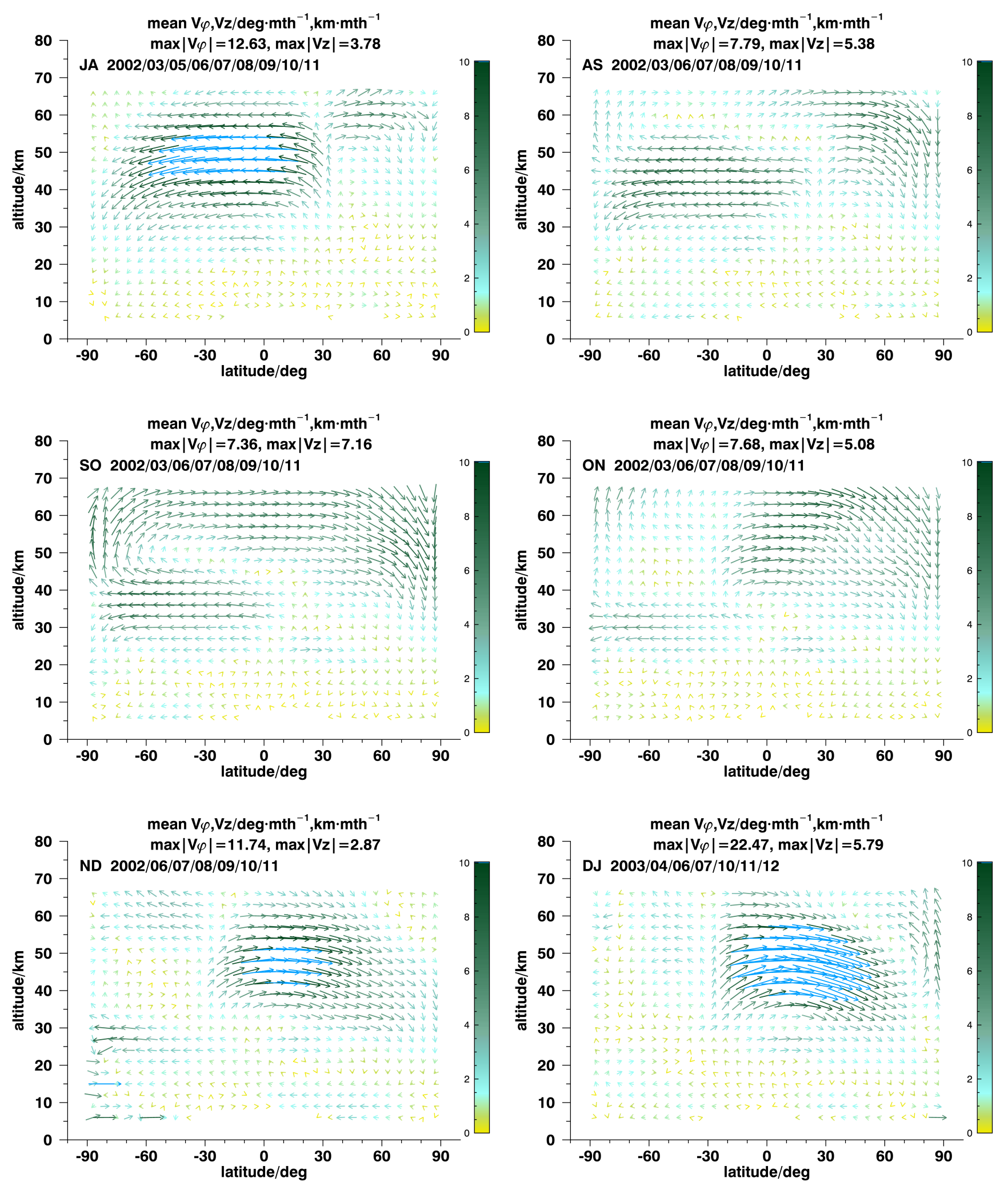

Figure S2: Mean monthly circulation patterns from July-August (top left, JA) to December-January (bottom right, DJ). For details, see Fig. S1. 

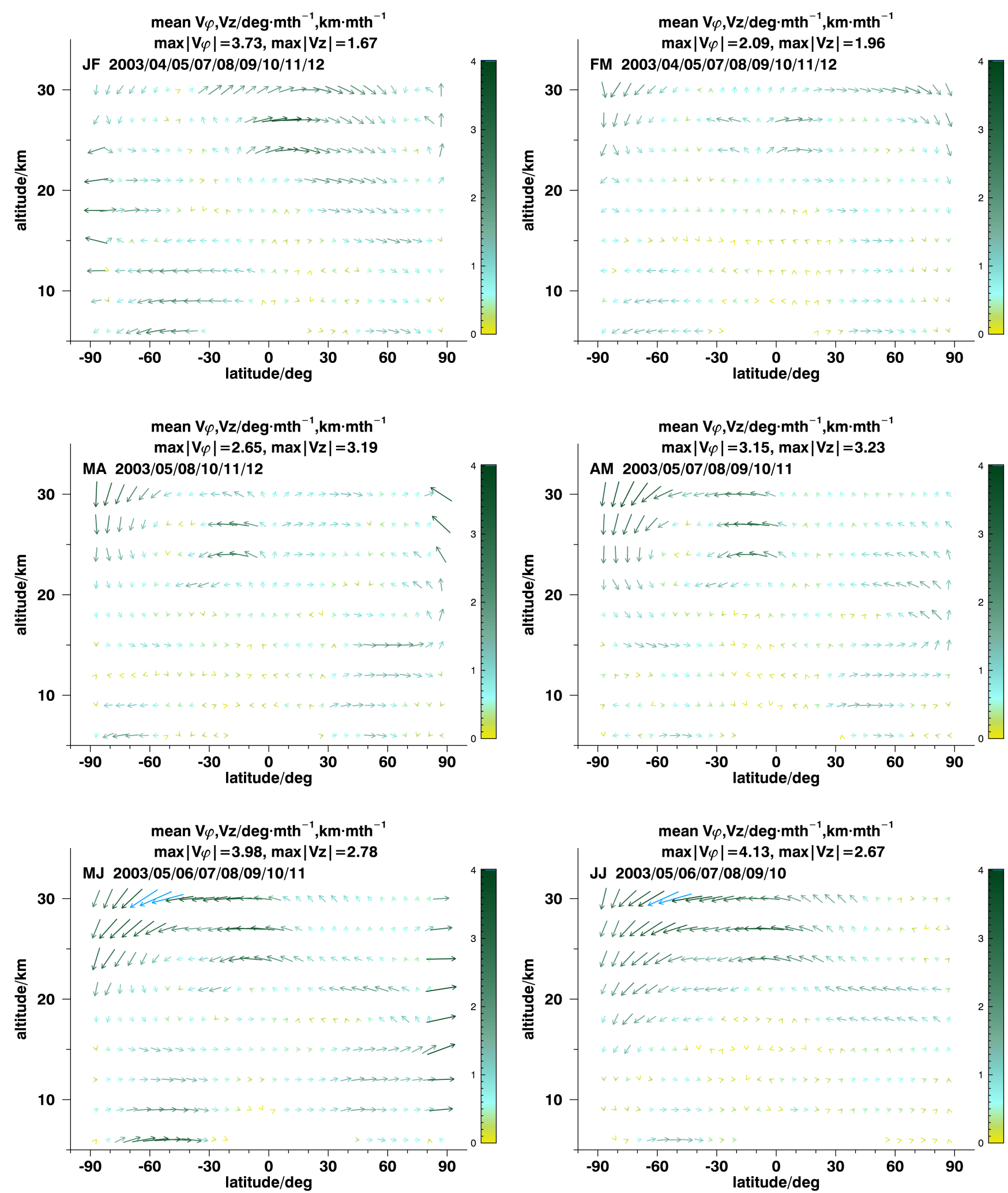

Figure S3: Same as Fig. S1 but for altitudes up to $30 \mathrm{~km}$ only. 

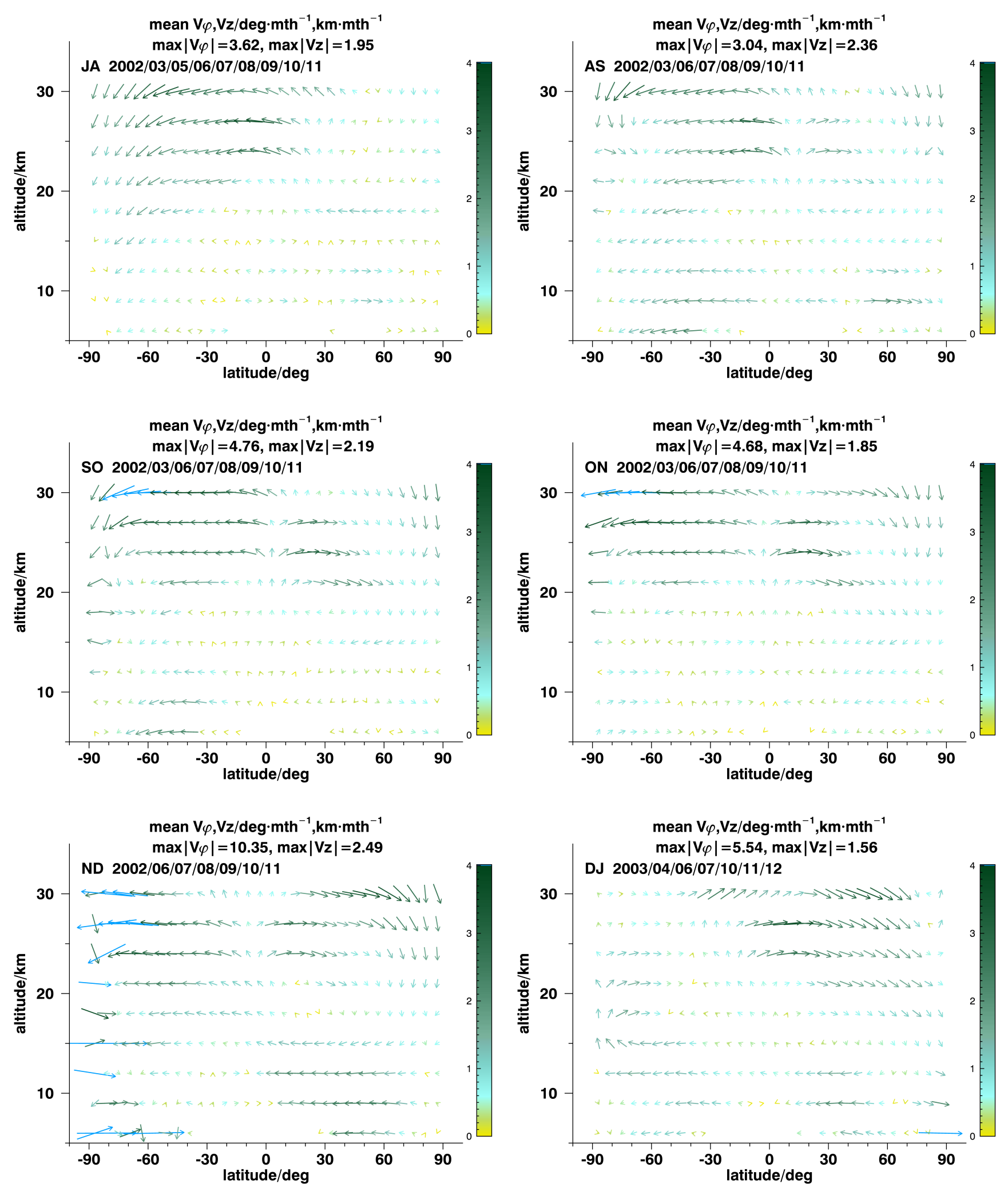

Figure S4: Same as Fig. S2 but for altitudes up to $30 \mathrm{~km}$ only. 

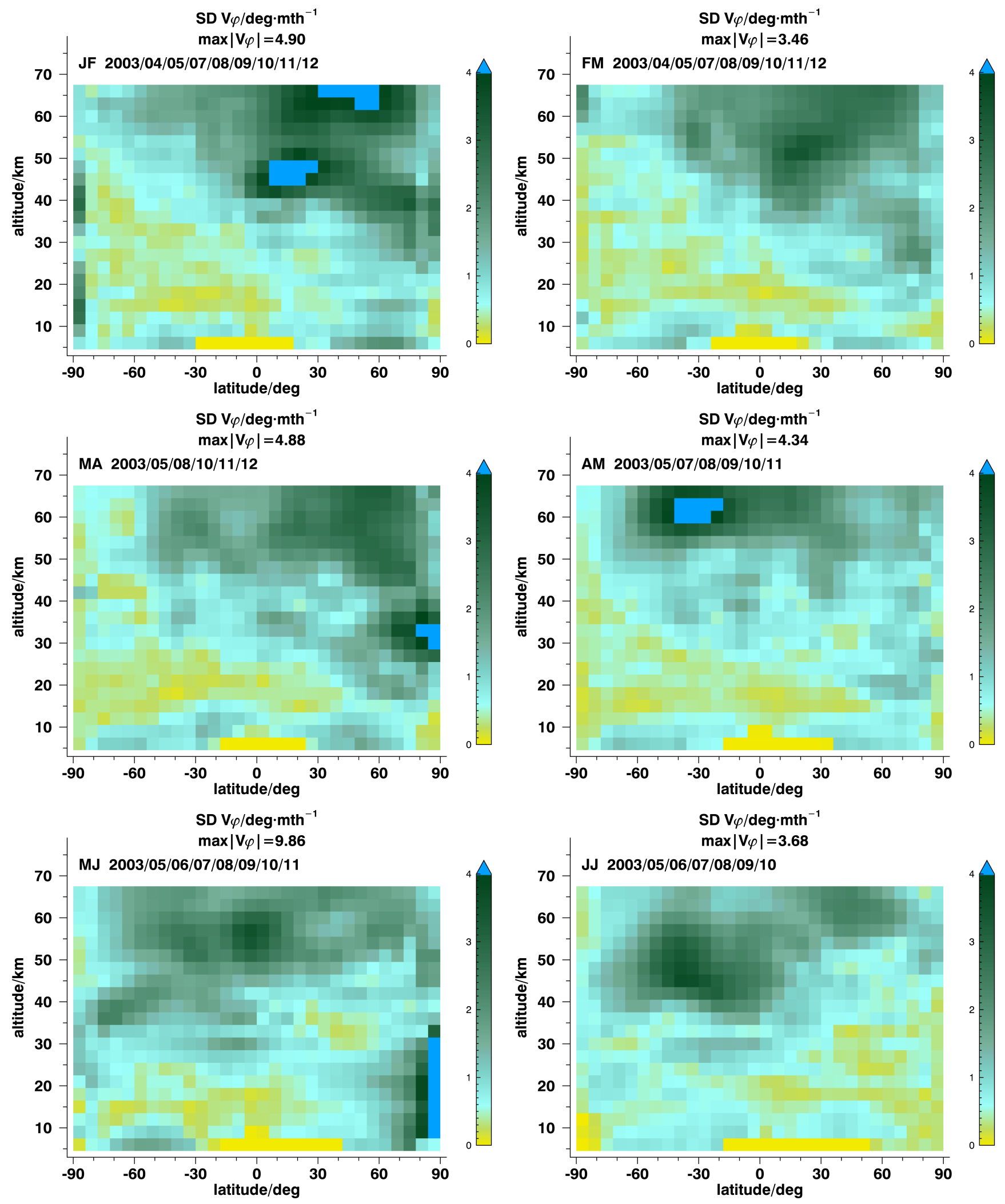

Figure S5: Inter-annual variability of the middle atmospheric meridional effective velocities in terms of sample standard deviations from January-February (top left, JF) to June-July (bottom right, JJ). 

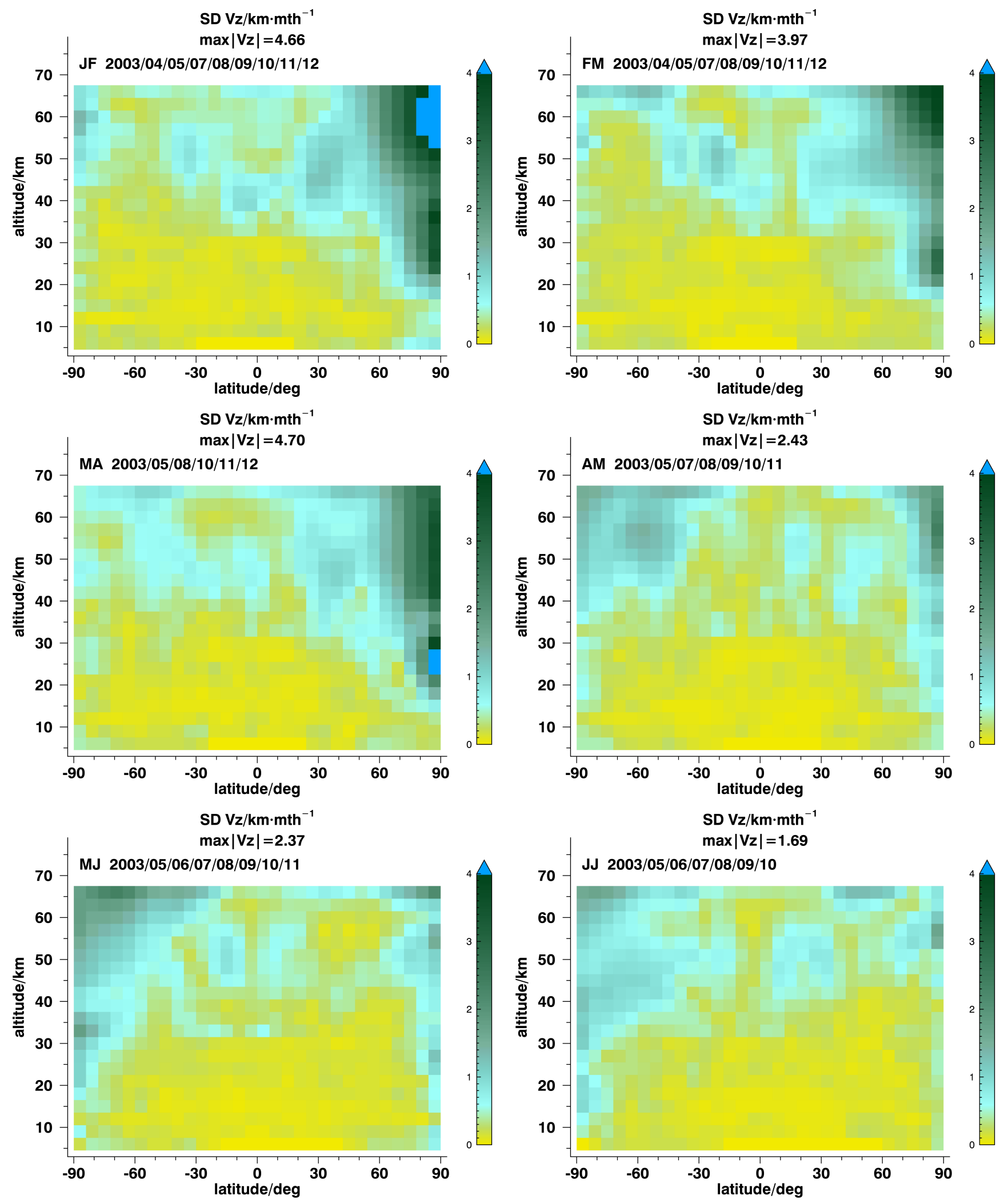

Figure S6: Inter-annual variability of the middle atmospheric vertical effective velocities in terms of sample standard deviations from January-February (top left, JF) to June-July (bottom right, JJ). 

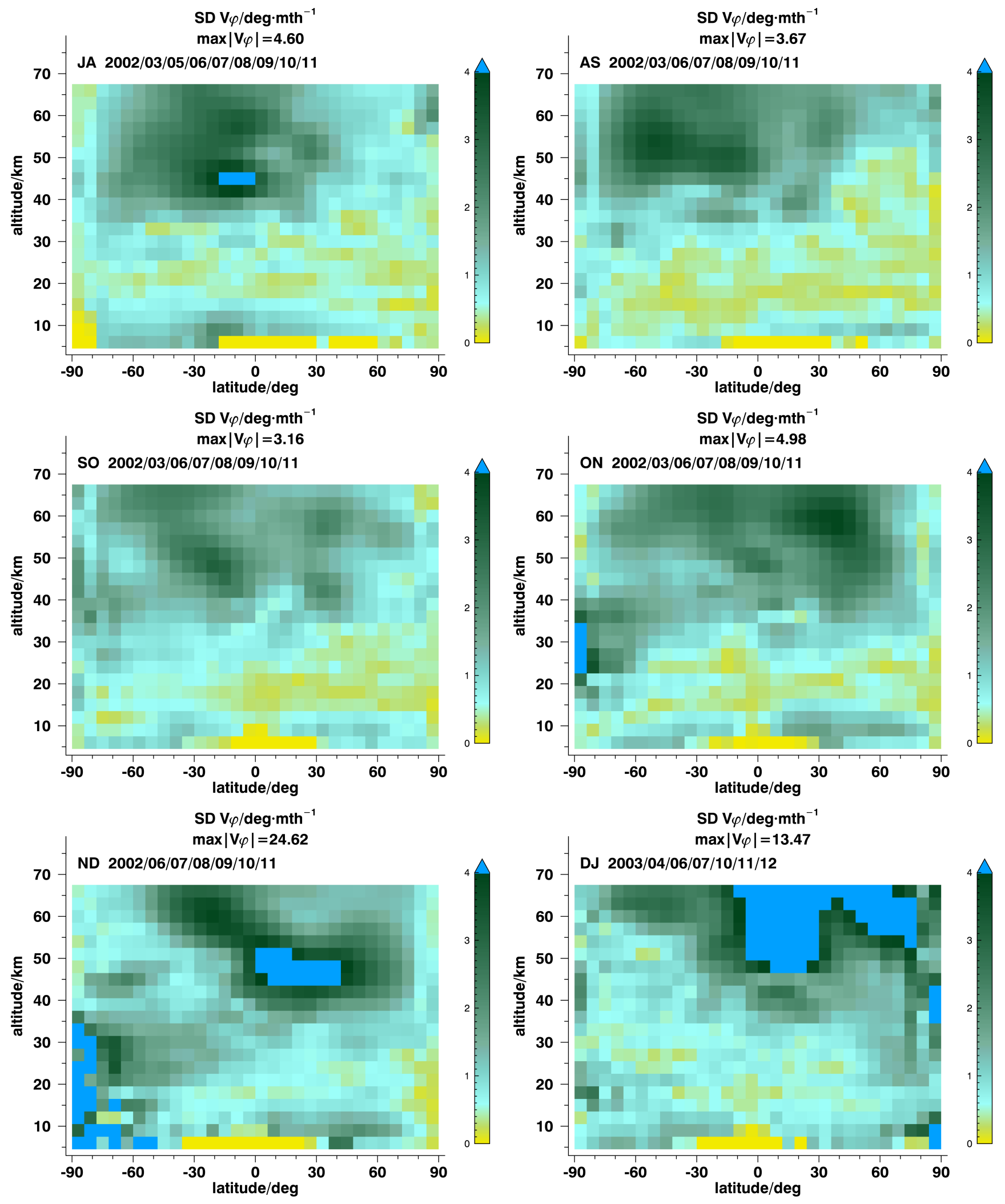

Figure S7: Inter-annual variability of the middle atmospheric meridional effective velocities in terms of sample standard variations from July-August (top left, JA) to December-January (bottom right, DJ). 

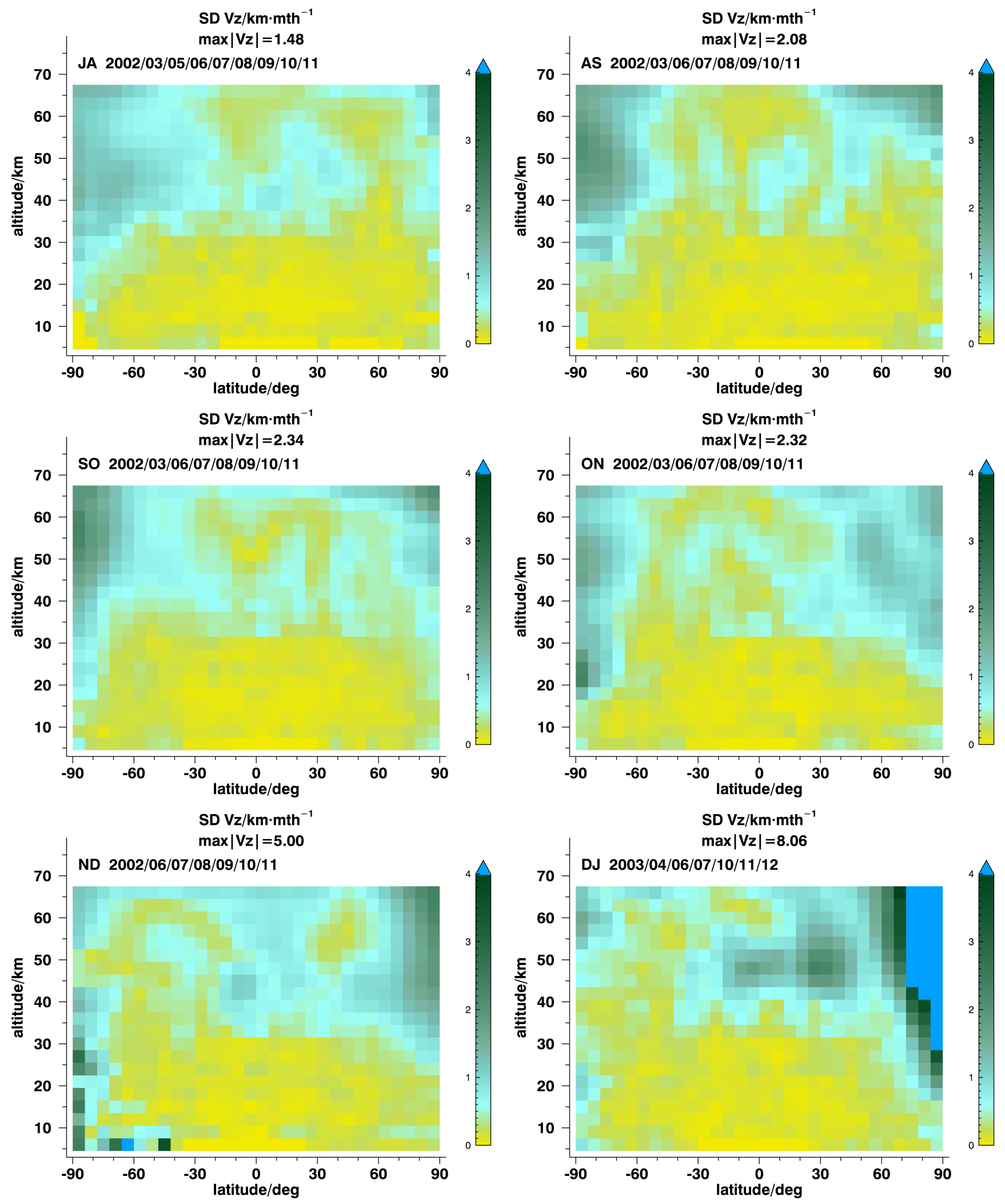

Figure S8: Inter-annual variability of the middle atmospheric vertical effective velocities in terms of sample standard variations from July-August (top left, JA) to December-January (bottom right, DJ). 